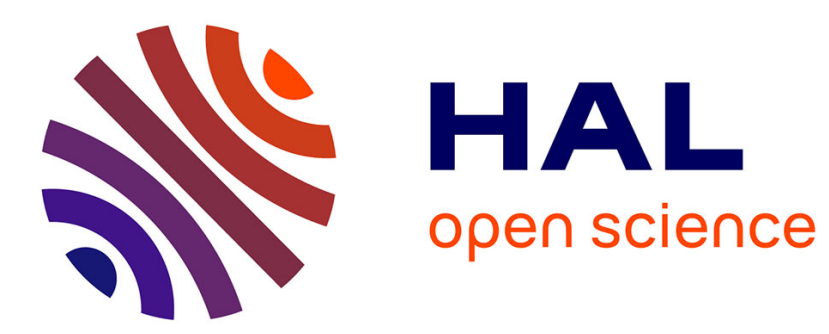

\title{
Analytical Modeling of Downlink CoMP in LTE-Advanced
}

Ahlem Khlass, Thomas Bonald, Salah Eddine Elayoubi

\section{To cite this version:}

Ahlem Khlass, Thomas Bonald, Salah Eddine Elayoubi. Analytical Modeling of Downlink CoMP in LTE-Advanced. VTC, 2015, Glasgow, United Kingdom. 10.1109/VTCSpring.2015.7145704 . hal01244192

\section{HAL Id: hal-01244192 \\ https://hal.inria.fr/hal-01244192}

Submitted on 15 Dec 2015

HAL is a multi-disciplinary open access archive for the deposit and dissemination of scientific research documents, whether they are published or not. The documents may come from teaching and research institutions in France or abroad, or from public or private research centers.
L'archive ouverte pluridisciplinaire HAL, est destinée au dépôt et à la diffusion de documents scientifiques de niveau recherche, publiés ou non, émanant des établissements d'enseignement et de recherche français ou étrangers, des laboratoires publics ou privés. 


\section{Analytical Modeling of Downlink CoMP in LTE-Advanced}

\author{
Ahlem Khlass \\ Orange Labs \& Telecom ParisTech \\ France \\ ahlem.khlass@orange.com
}

\author{
Thomas Bonald \\ Telecom ParisTech \\ Paris, France \\ thomas.bonald@telecom-paristech.fr
}

\author{
Salah Eddine Elayoubi \\ Orange Labs \\ Issy-Les-Moulineaux, France \\ salaheddine.elayoubi@orange.com
}

\begin{abstract}
In this paper, we discuss several Coordinated MultiPoint (CoMP) schemes proposed for LTE-Advanced systems. We investigate their benefits in a multi-antenna beamforming system where multiple cells may share their resources and jointly coordinate their transmissions to improve the performance at cell edge and the overall system capacity. We evaluate the system performance by combining flow-level analysis with numerical results from LTE-Advanced network simulator. We show that the intra-site coordination brings significant gains in beamforming systems, especially with the joint transmission scheme where the user throughput and the system capacity are improved.

Index Terms-Coordinated Multi-point transmission, LTEAdvanced, Flow-level modeling, Queuing theory, Simulations.
\end{abstract}

\section{INTRODUCTION}

LTE-Advanced is the current development trend of mobile communications, with a performance in terms of peak data rates expected to reach the values of $1 \mathrm{~Gb} / \mathrm{s}$ in downlink and $500 \mathrm{Mbit} / \mathrm{s}$ in uplink. This is enabled with the introduction of advanced radio technologies such as enhanced multiple-input multiple-output transmission techniques. However, inter-cell interference remains a very limiting factor, especially in the context of high resource reuse and complex cellular structures.

Several interference mitigation techniques have been investigated in order to overcome this problem of inter-cell interference [1]. A promising candidate that is investigated in this paper is the Coordinated multi-point (CoMP) transmission technique [2]. The CoMP feature has been introduced in release 11 of LTE/LTE-Advanced systems [3] in order to improve cell-edge throughput and system capacity. It can be seen as an evolution of multiple-input multiple-output (MIMO) technique which enables multiple points to share their antennas and to coordinate with each other in such a way that the transmission signals from/to other points do not interfere or can even be converted into a useful signal, especially at the cell edge where performance may be degraded [4], [5].

The CoMP transmission technique has been the focus of many studies in 3GPP for LTE-Advanced networks (e.g. [6] and references therein). In [7], several deployment scenarios and operational challenges were addressed. System-level performance has been conducted in [8]. Although such finite buffer simulations provide useful insights into the efficiency of coordination schemes, they are computationally intensive, especially when stability issues are addressed. Hence, the analytical methods are more interesting, especially when considering dynamic settings with flow arrivals and departures.

In this work, we analyze the flow level performance of the downlink CoMP transmission technique. We estimate the potential benefits on average and at cell edge in comparison to a classical transmission without cell coordination. This paper is organized as follows. First, we introduce the principle of CoMP transmissions in section II. In section III, we present the network layout. We introduce in section IV the blind beamforming system as a reference; then we provide flow-level analysis of the coordinated beamforming, basic and enhanced joint transmission schemes in sections V, VI and VII, respectively. Performance results under a variety of realistic network settings are provided in section VIII. Finally, conclusions and further work are drawn in section IX.

\section{PRINCIPLE}

The main idea of CoMP is as follows: when a user is in the cell-edge region, it may receive several strong signals from neighboring cells in addition to that from the serving cell. Given that, if the transmitted signals are coordinated by either silencing some or letting simultaneous transmissions, the downlink performance can be increased significantly. Indeed, this coordination enables to reduce interference or even to convert it into a useful signal in order to improve not only the users' experience but also the overall downlink system performance.

Two main techniques are possible for downlink CoMP: Coordinated beamforming/scheduling and Joint processing/transmission.

- Coordinated beamforming/scheduling (CB/CS) : The scheduling and beamforming decisions to serve one or multiple users are taken within a CoMP set (i.e. the cells involved into the CoMP transmission). However, the data is only available and transmitted by a single cell (i.e. with best channel conditions).

- Joint processing/transmission (JP/JT): The idea of joint processing/transmission is described by the the simultaneous transmission of data from multiple cells to a single user on the same time/frequency resource. This technique is similar to the multi-flow transmission proposed for HSPA+ systems [9]. 


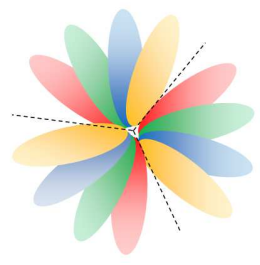

Fig. 1. System model.

These schemes have high potential to improve users' experience and the overall system capacity by reducing interference and increasing the signal strength. However, implementation issues are still constraining [7] (e.g. due to feedback requirements for inter-site CoMP ).

\section{NETWORK LAYOUT}

We consider a homogeneous single-layer beamforming system. We assume that each BS can provide $N$ spaced beams per sector (i.e. fixed codebook) and that each MS associates to its preferred beam that provides the best signal (i.e. maximum received power). An illustration is given in figure 1 with $N=4$ beams and $K=3$ sectors.

Note that it is out of our scope to investigate the optimal beamforming weights as done in several studies for downlink CoMP (e.g. [10] and references therein).

We propose in the following a flow-level analysis of intrasite coordination using coordinated beamforming and joint transmission schemes where there are no concerns about data availability or signaling overhead and latency between cells, since all operations are done locally within the same site.

\section{BLIND BEAMFORMING}

As a reference, we start by investigating the blind beamforming (BB) system in the presence of $N$ serving beams per sector.

We consider only elastic traffic. We assume that data flows arrive according to a Poisson process of intensity $\hat{\lambda}_{k}$ in sector $k$. Each flow stays in the system as long as the corresponding data have not been successfully transmitted to the User Equipment (UE). Flow sizes are assumed to be independent and exponentially distributed with mean $\sigma$ bits, although all our results are approximately insensitive to the flow size distribution beyond the mean.

We consider an arbitrary set $\mathcal{C}$ of classes of UEs indexed by $i$ to reflect the different radio conditions (i.e., locations) in the considered site and $\mathcal{C}_{k}$ the subset relative to sector $k$ with $k \in\{1, . ., K\}$. All UEs of the same class have approximately the same location and thus the same serving BS and the same data rate when scheduled. We denote by $\tilde{C}_{n, k}$ the classes of users covered by beam $n$ of sector $k$ with $n \in\{1, . ., N\}$ in the presence of $N$ serving beams. Since class- $i$ flows arrive at rate $\lambda_{i}$, the corresponding load is:

$$
\rho_{i}=\frac{\lambda_{i} \sigma}{R_{i}}
$$

where $R_{i}$ denotes the transmission rate at class- $i$ with a beamforming system.

\section{A. Blind scheduling algorithm}

We consider a centralized scheduler per radio site. According to a blind beamforming system, the scheduler selects a user at random in each sector. This latter is served alone and blocks all other users in the corresponding sector.

Although such Round Robin-based scheduler is simple and does not take into account the radio channel conditions, it remains a good approximation of any opportunistic scheduler like proportional fair (e.g. [11]) when the channel variations are limited or too fast to be exploited.

\section{B. Flow-level analysis}

We denote by $B_{n, k}(t)$ the total number of users in beam $n$ of sector $k$ at time $t$ and $\vec{B}(t)$ the corresponding vector. Whenever $\vec{B}(t)=\vec{b}$, the scheduler selects a user in beam $n$ of sector $k$ a fraction of time of:

$$
\phi_{n, k}(\vec{b})=\frac{b_{n, k}}{\left|b_{k}\right|},
$$

where $\left|b_{k}\right|$ denotes the total number of users in sector $k$.

The arrival rate per beam $\tilde{\lambda}_{n, k}$ is expressed by

$$
\tilde{\lambda}_{n, k}=\sum_{i \in \tilde{\mathcal{C}}_{n, k}} \lambda_{i}
$$

and the harmonic mean transmission rate:

$$
\tilde{R}_{n, k}=\frac{\sum_{i \in \tilde{\mathcal{C}}_{n, k}} p_{i}}{\sum_{i \in \tilde{\mathcal{C}}_{n, k}} \frac{p_{i}}{R_{i}}},
$$

with

$$
\sum_{i \in \mathcal{C}_{k}} p_{i}=\sum_{i \in \mathcal{C}_{k}} \lambda_{i} / \hat{\lambda}_{k}=1
$$

The queuing system is described by the following transition rates of the Markov process $\vec{B}(t)$ from state $\vec{b}$ to state $\vec{b}^{\prime}$ :

$$
q\left(\vec{b}, \vec{b}^{\prime}\right)= \begin{cases}\tilde{\lambda}_{n, k} & \text { for } \vec{b}^{\prime}=\vec{b}+\vec{e}_{n, k}, \\ \phi_{n, k}(\vec{b}) \frac{\tilde{R}_{n, k}}{\sigma} & \text { for } \vec{b}^{\prime}=\vec{b}-\vec{e}_{n, k},\end{cases}
$$

where $\vec{e}_{n, k}$ denotes the unit vector on component $n$, for $n \in$ $\{1, \ldots, N\}$ and $k \in\{1, . ., K\}$.

The system corresponds to three independent multi-class processor sharing queues with total load $\hat{\rho}_{k}=\sum_{n=1}^{N} \tilde{\rho}_{n, k}$ where

$$
\tilde{\rho}_{n, k}=\frac{\tilde{\lambda}_{n, k} \sigma}{\tilde{R}_{n, k}} .
$$

Under the stability condition in each sector expressed by $\forall k \in \mathcal{K}, \quad \hat{\rho}_{k}<1$, we deduce the stationary distribution describing the number of flows in the system:

$$
\pi(\vec{b})=\prod_{k=1}^{K}\left(1-\hat{\rho}_{k}\right) \frac{\left|b_{k}\right| !}{\prod_{n=1}^{N} b_{n, k} !} \prod_{n} \tilde{\rho}_{n, k}^{b_{n, k}},
$$

Using Little's law, we derive the mean duration $\tau_{i}$ of class- $i$ flows:

$$
\tau_{i}=\frac{E\left(b_{n, k}\right)}{\lambda_{i}} .
$$


With the mean number of class- $i$ flows given by:

$$
E\left(b_{n, k}\right)=\frac{\rho_{i}}{1-\hat{\rho}_{k}}
$$

we deduce the flow throughput (in bit/s) in beam $n$ of sector $k$ defined as the radio of the mean flow size (in bits) to the mean flow duration (in s):

$$
\gamma_{n, k}=\tilde{R}_{n, k}\left(1-\hat{\rho}_{k}\right),
$$

Then the mean throughput in the sector $k$ is given by:

$$
\hat{\gamma}_{k}=\hat{R}_{k}\left(1-\hat{\rho}_{k}\right)
$$

\section{COORDINATED BEAMFORMING}

When coordinated beamforming is enabled, users are selected under additional constraints in order to avoid high interference states caused by serving cell-edge users.

\section{A. Fixed coordinated beamforming}

A way of ensuring less intra-site interference is by activating the same beam in each sector. The scheduler selects a user in beam $n$ of sector $k$ a fraction of time of:

$$
\phi_{n, k}(\vec{b})=\frac{\sum_{k=1}^{K} b_{n, k}}{|b|}
$$

where $|b|$ denotes the total number of users in the whole site.

The system corresponds to a set of $N \times K$ coupled processor-sharing queues with state-dependent service rates. The queuing system can be described by a Markov process whose transition rates are given by:

$$
q\left(\vec{b}, \vec{b}^{\prime}\right)= \begin{cases}\tilde{\lambda}_{n, k} & \text { for } \vec{b}^{\prime}=\vec{b}+\vec{e}_{n, k}, \\ \phi_{n, k}(\vec{b}) \frac{\tilde{R}_{n, k}^{\prime}}{\sigma} & \text { for } \vec{b}^{\prime}=\vec{b}-\vec{e}_{n, k},\end{cases}
$$

where $\tilde{R}_{n, k}^{\prime}$ refers to the transmission rate in beam $n$ of sector $k$ when coordinated beamforming is performed.

This Markov process is reversible and equivalently, the queuing system corresponds to a Whittle network. The stationary distribution of the Markov process $\vec{B}(t)$ is given by:

$$
\pi(\vec{b})=\pi(\overrightarrow{0})\left(\sum_{k=1}^{K} b_{1, k}, \ldots, \sum_{k=1}^{K} b_{N, k}\right) \prod_{k=1}^{3} \prod_{n=1}^{N} \tilde{\rho}_{n, k}^{b_{n, k}},
$$

under the stability condition verified in each sector $k$ :

$$
\sum_{n=1}^{N} \tilde{\rho}_{n, k}<1
$$

where $\tilde{\rho}_{n, k}$ denotes the load per beam defined in (2).

For illustration purpose, we restrict the number of beams to $N=2$ per sector and we suppose that all beams have the same load such that $\tilde{\rho}_{n}=\tilde{\rho}, \quad \forall n \in\{1, . ., N\}$. We deduce the stationary distribution of the Markov process such that:

$$
\pi(0)=\frac{(-1+2 \tilde{\rho})^{5}}{\left(1-4 \tilde{\rho}+10 \tilde{\rho}^{2}-12 \tilde{\rho}^{3}+6 \tilde{\rho}^{4}\right)} .
$$

The flow throughput of users covered by beam $n$ is then given by:

$$
\tilde{\gamma}_{n}=\tilde{R}_{n}^{\prime} \frac{\left(1-6 \tilde{\rho}+18 \tilde{\rho}^{2}-32 \tilde{\rho}^{3}+30 \tilde{\rho}^{4}-12 \tilde{\rho}^{5}\right)}{3-6 \tilde{\rho}+12 \tilde{\rho}^{2}-12 \tilde{\rho}^{3}+6 \tilde{\rho}^{4}}
$$

\section{B. Flexible coordinated beamforming scheme}

A more advanced coordinated beamforming scheme will avoid high interference states while optimizing the scheduling opportunities. Indeed, with a flexible scheme, a minimum separation between active beams is ensured without having a fixed scheduling scheme. Accordingly, the scheduler selects a user in beam $n$ of sector $k$ a fraction of time of:

$$
\begin{gathered}
\phi_{1, k}(\vec{b})=\frac{b_{1, k}}{|b|}+\frac{|b|-\left|b_{k}\right|-b_{N, k-1}}{|b|} \times \frac{b_{1, k}}{\left|b_{k}\right|}, \\
\phi_{N, k}(\vec{b})=\frac{b_{N, k}}{|b|}+\frac{|b|-\left|b_{k}\right|-b_{N, k+1}}{|b|} \times \frac{b_{N, k}}{\left|b_{k}\right|}, \\
\phi_{n, k}(\vec{b})=\frac{b_{n, k}}{\left|b_{k}\right|}, \quad \forall n \in\{1, . ., N\} \backslash\{1, N\},
\end{gathered}
$$

The system corresponds to a set of $N \times K$ coupled processor-sharing queues with state-dependent service rates. The queuing system can be described by the Markov process whose transition rates are given in (6) by replacing the fractions of time $\phi_{n, k}$ by their corresponding values. This Markov process is no more reversible. Therefore, the stationary distribution of the number of active flows can not be derived analytically. The performance of such process is deduced numerically by resolution of the corresponding Markov process.

\section{BASIC JOINT TRANSMISSION SCHEME}

The basic joint transmission technique enables a user in the overlapping area between two cells to be jointly and simultaneously served by both.

We suppose that the site is divided into six zones:

- Non-coordination zones where users are served exclusively by a unique $B S$ noted $Z_{1}, Z_{2}$ and $Z_{3}$.

- Coordination zones where users are served simultaneously by two $B S$ s noted $Z_{12}, Z_{23}$ and $Z_{31}$.

We denote by $\overline{\mathcal{C}}_{12} \subset \mathcal{C}$ the set of classes in zone 12 ; UEs of class $i \in \overline{\mathcal{C}}_{12}$ are served simultaneously by BS 1 and 2 at rate $R_{i}^{\prime}$. The sets $\overline{\mathcal{C}}_{1}=\mathcal{C}_{1} \backslash \overline{\mathcal{C}}_{12}$ and $\overline{\mathcal{C}}_{2}=\overline{\mathcal{C}}_{2} \backslash \overline{\mathcal{C}}_{12}$ define zones 1 and 2 of sectors 1 and 2, respectively, where UEs are not subject to coordination; UEs of class $i \in \overline{\mathcal{C}}_{j}$ are served by BS $j$ at rate $R_{i}$, for $j \in\{1,2\}$.

Let $\vec{Z}(t)$ be the vector of the number of flows in each zone. When $\vec{Z}(t)=\vec{z}$, the considered scheduler selects UEs in zone 1 a fraction of time:

$$
\phi_{1}(\vec{z})=\frac{z_{1}+z_{23}}{|z|}+\frac{z_{2}}{|z|} \frac{z_{1}+z_{3}}{z_{1}+z_{3}+z_{31}}+\frac{z_{3}}{|z|} \frac{z_{1}+z_{2}}{z_{1}+z_{2}+z_{12}}
$$

and UEs in zone 12 a fraction of time:

$$
\phi_{12}(\vec{z})=\frac{z_{12}}{|z|}+\frac{z_{3}}{|z|} \frac{z_{12}}{z_{1}+z_{2}+z_{12}},
$$


where $|z|$ denotes the total number of users in the whole site. We obtain (9) for instance by observing that a UE in zone 12 is either selected first by the scheduling algorithm, with probability $z_{12} /|z|$, or after the selection of a UE in zone 3, with probability $z_{3} /|z|$; in the latter case, only UEs in zones 1,2 or 12 can be selected in the same timeslot. Expression (8) can be derived in the same way, and the timeslot allocation in the other zones follow by symmetry.

The system corresponds to a set of six coupled processorsharing queues with state-dependent service rates. The analytical performance in terms of flow throughput can be deduced from our previous work on multi-flow transmission for HSPA+ systems [12]. We obtain:

$$
\begin{gathered}
\forall n, \tilde{\mathcal{C}}_{n} \subset \mathcal{C}_{1}, \quad \tilde{\gamma}_{n} \approx \tilde{R}_{n}\left(1-\bar{\rho}_{1}-\bar{\rho}_{31}-\bar{\rho}_{12}\right) \\
\forall n, \tilde{\mathcal{C}}_{n} \subset \mathcal{C}_{12}, \\
\tilde{\gamma}_{n} \approx \tilde{R}_{n}^{\prime} \frac{\left(1-\bar{\rho}_{12}\right)}{1+\frac{\bar{\rho}_{1}+\bar{\rho}_{31}}{1-\bar{\rho}_{1}-\bar{\rho}_{12}-\bar{\rho}_{31}}+\frac{\bar{\rho}_{2}+\bar{\rho}_{23}}{1-\bar{\rho}_{2}-\bar{\rho}_{12}-\bar{\rho}_{23}}}
\end{gathered}
$$

under the stability condition:

$$
\begin{aligned}
& \bar{\rho}_{1}+\bar{\rho}_{12}+\bar{\rho}_{31}<1, \\
& \bar{\rho}_{2}+\bar{\rho}_{12}+\bar{\rho}_{23}<1, \\
& \bar{\rho}_{3}+\bar{\rho}_{31}+\bar{\rho}_{23}<1 .
\end{aligned}
$$

Note that $\tilde{R}_{n}^{\prime}$ denotes the transmission rate in beam $n$ when joint transmission is performed.

\section{ENHANCED JOINT TRANSMISSION SCHEME}

Considering an enhanced scheme where coordination is only performed when resources are available. Indeed, each sector can transmit data to a cell-edge user located in an adjacent sector while scheduling its own users. This means that only beams involved in the JT will be blocked and leaves the possibility for another user to be scheduled in a given sector.

We consider the same system model and notations except that we now differentiate between zone 12, consisting of classes in the coordination region served by BS 1, and zone 21 , consisting of classes in the coordination region served by BS 2, and so on for all the coordination zones. We denote by $z_{j}$ the number of users in zone $j$ for $j \in$ $\{12,1,21,2,23,3,32,13,31\}$ and by $y_{k}$ the total number of users in sector $k . \vec{Z}$ and $\vec{Y}$ refer to their corresponding vectors, respectively.

According to the enhanced JT scheme, the service in each coordination zone depends on the state of the other zones. We propose to decouple the system by replacing the statedependent service rates in each zone by their mean. Accordingly, the system behaves as three independent multi-class processor-sharing queues. The queuing system is described by the following transition rates of the Markov process $\vec{Z}(t)$

$$
q\left(\vec{z}, \vec{z}^{\prime}\right)= \begin{cases}\bar{\lambda}_{j} & \text { for } \vec{z}^{\prime}=\vec{z}+\vec{e}_{j}, \forall j \\ \phi_{j}(\vec{z}) \frac{\bar{R}_{j}}{\sigma} & \text { for } \vec{z}^{\prime}=\vec{z}-\vec{e}_{j}, j=\{1,2,3\} \\ \phi_{j}(\vec{z}) \frac{\bar{\beta} \bar{R}_{j}}{\sigma} & \text { for } \vec{z}^{\prime}=\vec{z}-\vec{e}_{j}, \forall j \backslash\{1,2,3\}\end{cases}
$$

with $\phi_{j}(\vec{z})=\frac{z_{j}}{y_{k}}$, and where $\bar{\lambda}_{j}$ and $\bar{R}_{j}$ denote the arrival rate and the transmission rate per zone $j$. The term $\bar{\beta}$ refers the coordination gain obtained by averaging over all possible states of neighboring sectors, it is expressed in zone 12 by :

$$
\begin{aligned}
\bar{\beta}_{12} & =\frac{\bar{R}_{12}^{\prime}}{\bar{R}_{12}}\left[P\left(Y_{2}=0\right)+E\left(\frac{Z_{23}}{Y_{2}} \mid Y_{2}>0\right)\right]+E\left(\frac{Z_{21}}{Y_{2}} \mid Y_{2}>0\right) \\
& +\frac{1}{2}\left(1-\frac{\bar{R}_{12}^{\prime}}{\bar{R}_{12}}\right) E\left(\frac{Z_{32}}{Y_{3}} \mid Y_{3}>0\right)\left[P\left(Y_{2}=0, Y_{3}>0\right)\right. \\
& \left.+E\left(\frac{Z_{2}}{Y_{2}} \mid Y_{2}>0\right)\right],
\end{aligned}
$$

and so on for the other zones. Indeed, a UE in zone 12 benefits from coordination if both sector 2 and sector 3 are empty. If sector 2 is empty and UEs in zones 12 and 32 are scheduled, we assume that BS 2 selects to coordinate users in zone 12 or in zone 32 uniformly at random, hence the factor $\frac{1}{2}$. However, when sector 2 is not empty, coordination can be performed when a UE in zone 32 is scheduled or when a UE in zone 2 is scheduled and BS 2 selects to coordinate UEs in zone 12.

\section{NUMERICAL RESULTS}

We present in this section a numerical analysis where the developed models take as input realistic rates from an LTEadvanced system simulation.

TABLE I

SIMULATION PARAMETERS

\begin{tabular}{|l|c|}
\hline Parameter & Value \\
\hline \hline Site layout & 19 hexagonal tri-sector sites \\
\hline Inter-site distance & $500 \mathrm{~m}$ \\
\hline Cell radius & $350 \mathrm{~m}$ \\
\hline System bandwidth & $10 \mathrm{MHz}$ \\
\hline Carrier frequency band & $2.6 \mathrm{GHz}$ \\
\hline Propagation environment & urban coverage \\
\hline Propagation model & Hata model \\
\hline Path loss exponent & 1 \\
\hline Number of antenna at terminals & 4 \\
\hline Number of antennas per sector & Linear array, $\lambda / 2$ \\
\hline Antenna configuration and spacing & $G(\theta)-\min \left[12\left(\frac{\theta}{\theta_{3 d B}}{ }^{2}, A_{m}\right)\right.$ \\
\hline $\begin{array}{l}\text { Antenna gain model } \\
\text { (3GPP model) }\end{array}$ & $\theta_{3 d B}=30^{\circ}, A_{m}=20$ \\
\hline
\end{tabular}

\section{A. Achievable rates with intra-site coordination}

Considering the system parameters summarized in table I, we calculate the peak transmission rates (i.e. stand-alone throughput) achievable at different positions of the reference site on the basis of 100 independent network states using the Shannon formula given by:

$$
R_{i}=W \log \left(1+S I N R_{i}\right),
$$

with $W$ denotes the system bandwidth. The transmission rates are given in table II and table III under several intra-site coordination schemes and in two network settings: i) a network with ideal beams and ii) a network with non-ideal beams where the direction of each beam is perturbed uniformly at random of $\left[-25^{\circ}, 25^{\circ}\right]$ around the original direction.

We first note that the blind beamforming enables to achieve higher transmission rates compared to a classical system without beamforming since the transmitted energy of such system 
TABLE II

PEAK DATA RATES (IN MBIT/S) WITH IDEAL BEAMS

\begin{tabular}{|l|c|c|}
\hline Scenario & Average rate & Cell edge rate \\
\hline Without beamforming & 3.14 & 1.29 \\
\hline Blind beamforming & 6.24 & 2.17 \\
\hline Fixed coordinated beamforming & 6.27 & 2.17 \\
\hline Flexible coordinated beamforming & 6.28 & 2.19 \\
\hline Basic joint transmission & 6.36 & 2.19 \\
\hline Enhanced joint transmission & 6.25 & 2.46 \\
\hline
\end{tabular}

TABLE III

PEAK DATA RATES (IN MBIT/S) WITH NON-IDEAL BEAMS

\begin{tabular}{|l|c|c|}
\hline Scenario & Average rate & Cell edge rate \\
\hline Without beamforming & 2.71 & 1.05 \\
\hline Blind beamforming & 5.67 & 2.25 \\
\hline Fixed coordinated beamforming & 6.16 & 2.44 \\
\hline Flexible coordinated beamforming & 6.22 & 2.46 \\
\hline Basic joint transmission & 6.32 & 2.44 \\
\hline Enhanced joint transmission & 6.25 & 2.46 \\
\hline
\end{tabular}

is confined into directional beams. We see also that the gains of intra-site coordination either with coordinated beamforming or joint transmission are too limited in a network with ideal beams since the intra-site interference is naturally avoided with ideal beams. However, in a network with non-ideal beams, we see that the coordinated beamforming either with a fixed or a flexible scheme enables to achieve an improvement of $10 \%$ in transmission rates compared to the blind beamforming since the intra-site interference is better controlled. With the basic JT scheme, the peak rates are improved by $12 \%$ on average and $20 \%$ at cell edge. The enhanced JT achieves less data rates compared to the basic JT due to the presence of a second user that shares the same resource.

\section{B. Performance results}

For various traffic intensities, we plot in figure 2 the performance of several CoMP schemes. Results are obtained by simulation of $10^{6}$ jumps of their corresponding Markov process in terms of mean throughput.

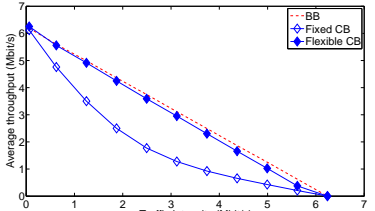

(a) With ideal beams

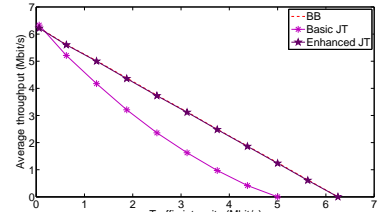

(c) With ideal beams

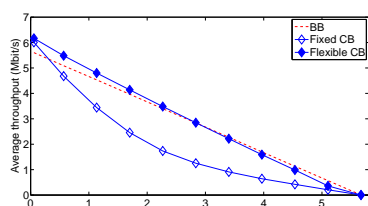

(b) With non-ideal beams

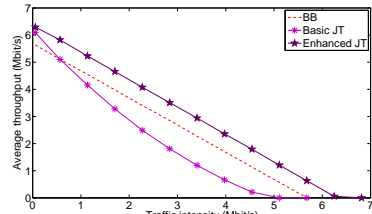

(d) With non-ideal beams

Fig. 2. Mean throughput vs. traffic intensities with fully loaded neighbors.

The first observation we can make is that there are no gains from intra-site coordination in a network with ideal beams since the intra-site interference is naturally avoided. However, with non-ideal beams, we notice that the performance gains of

intra-site coordinated beamforming are limited in comparison to a blind beamforming system. The flexible coordinated beamforming enables to better improve the average throughput, especially at low loads, by avoiding to serve two celledge users. Besides, we see that the basic joint transmission enables to achieve higher throughput at low loads, but makes the system instable at high loads. However, the enhanced joint transmission provides an improvement on average throughput and in system stability since each sector can coordinate its neighbors' transmissions without blocking its own users.

\section{CONCLUSiON}

Throughout this paper, we investigated several CoMP schemes by combining flow-level analysis with network simulations. We evaluated the performance in terms of average user throughput achievable under different traffic intensities. Results with non-ideal beams showed that intra-site coordination achieves significant gains over the blind beamforming system, and particularly the enhanced joint transmission scheme which improves the throughput as well as the system stability since each sector can help its neighbors while serving its own users. This can not the case with ideal beams, since intra-site interference is naturally avoided.

As future work, we intend to investigate the performance of CoMP transmission in a multi-user environment with enhanced physical-layer considerations.

\section{REFERENCES}

[1] "Evolved universal terrestrial radio access (E-UTRA); further advancements for E-UTRA physical layer aspects (3GPP release9)."

[2] E. Hossain, T.-i. Kim, and V. K. Bhargava, Cooperative cellular wireless networks, vol. 38. Cambridge University Press Cambridge, MA, 2011.

[3] "3GPP TR 36.819: Technical specification group radio access network; coordinated multi-point operation for LTE physical layer aspects (release 11)," Dec. 2011

[4] P. Marsch and G. P. Fettweis, Coordinated Multi-Point in Mobile Communications: from theory to practice. Cambridge University Press, 2011.

[5] D. Gesbert, S. Hanly, H. Huang, S. Shamai Shitz, O. Simeone, and W. Yu, "Multi-cell mimo cooperative networks: A new look at interference," Selected Areas in Communications, IEEE Journal on, vol. 28, pp. 1380-1408, December 2010.

[6] M. Sawahashi, Y. Kishiyama, A. Morimoto, D. Nishikawa, and M. Tanno, "Coordinated multipoint transmission/reception techniques for lte-advanced," Wireless Communications, IEEE, vol. 17, pp. 26-34, June 2010.

[7] D. Lee, H. Seo, B. Clerckx, E. Hardouin, D. Mazzarese, S. Nagata, and K. Sayana, "Coordinated multipoint transmission and reception in LTE-advanced: deployment scenarios and operational challenges," IEEE Communications Magazine, vol. 50, no. 2, pp. 148-155, 2012.

[8] S. Brueck, L. Zhao, J. Giese, and M. A. Amin, "Centralized scheduling for joint transmission coordinated multi-point in lte-advanced," in Smart Antennas (WSA), 2010 International ITG Workshop on, pp. 177-184, IEEE, 2010

[9] “3GPP TR 25.872: technical specification group radio access network; high speed packet access (HSDPA) multipoint transmission,” Dec. 2011.

[10] H. Dahrouj and W. Yu, "Coordinated beamforming for the multicell multi-antenna wireless system," Wireless Communications, IEEE Transactions on, vol. 9, pp. 1748-1759, May 2010.

[11] W. Yu, T. Kwon, and C. Shin, "Multicell coordination via joint scheduling, beamforming, and power spectrum adaptation," Wireless Communications, IEEE Transactions on, vol. 12, pp. 1-14, July 2013.

[12] A. Khlass, T. Bonald, and S. E. Elayoubi, "Flow-Level performance of intra-site coordination in cellular networks," in IEEE Modeling and Optimization in Mobile, Ad Hoc and Wireless Networks (WiOpt), pp. 303-310, May. 2013. 